\title{
Cooperative Multihop Broadcast for Wireless Networks
}

\author{
Ivana Maric Member, IEEE and Roy D. Yates Member, IEEE
}

\begin{abstract}
We address the minimum-energy broadcast problem under the assumption that nodes beyond the nominal range of a transmitter can collect the energy of unreliably received overheard signals. As a message is forwarded through the network, a node will have multiple opportunities to reliably receive the message by collecting energy during each retransmission. We refer to this cooperative strategy as accumulative broadcast. We seek to employ accumulative broadcast in a large scale loosely synchronized, low-power network. Therefore, we focus on distributed network layer approaches for accumulative broadcast in which loosely synchronized nodes use only local information. To further simplify the system architecture, we assume that nodes forward only reliably decoded messages.

Under these assumptions, we formulate the minimum-energy accumulative broadcast problem. We present a solution employing two subproblems. First, we identify the ordering in which nodes should transmit. Second, we determine the optimum power levels for that ordering. While the second subproblem can be solved by means of linear programming, the ordering subproblem is found to be NP-complete. We devise a heuristic algorithm to find a good ordering. Simulation results show the performance of the algorithm to be close to optimum and a significant improvement over the well known BIP algorithm for constructing energy-efficient broadcast trees. We then formulate a distributed version of the acumulative broadcast algorithm that uses only local information at the nodes and has performance close to its centralized counterpart.
\end{abstract}

Index Terms-Minimum-energy broadcast, reliable forwarding, wideband regime, distributed algorithm.

\section{INTRODUCTION.}

In a wireless network, the objective of the minimum-energy broadcast problem is to broadcast data reliably to all network nodes at a given rate with minimum transmitted power. The problem of broadcasting in a wireless network has been researched extensively (see [1] and references therein). In [2], the minimum-energy broadcast problem was formulated as a minimum-energy broadcast tree problem. Although the minimum-cost broadcast tree can be found in $O\left(n^{2}\right)$ operations in a wired network [3], the equivalent wireless problem was shown in [4] to be NP-hard and later on, in [5]-[7] to be NP-complete. The greater difficulty of the wireless broadcast tree problem stems from the wireless multicast advantage [2], the fact that a wireless transmission can be received by all nodes in the transmission range. In [2], the authors proposed

Manuscript received July 15, 2003; revised January 31, 2004. This work was supported by New Jersey Commission on Science and Technology and NSF grant NSF ANI 0338805.

The authors are with Wireless Network Information Laboratory (WINLAB), Department of Electrical and Computer Engineering, Rutgers University, Piscataway, NJ 08854 USA (e-mail: ivanam@winlab.rutgers.edu; ryates@winlab.rutgers.edu). the Broadcast Incremental Power (BIP) algorithm, a greedy heuristic that uses the principle of Prim's algorithm [8] while assigning costs to the nodes in a way that exploits the wireless multicast advantage. Analytical results for the performance of BIP are given in [9]. Several other heuristics for constructing energy-efficient broadcast trees have been proposed in the literature and evaluated by simulations (see [4]-[7], [10] and references therein).

The wireless formulation of the minimum-energy broadcast problem assumes that a node can benefit from a certain transmission only if the received power is above a threshold required for reliable communication. This is a pessimistic assumption. When the received power is below the required threshold, but above the receiver noise floor, a node can collect energy from the unreliable reception of the sent information. For example, in a Bluetooth system [11], the nominal transmitted power is $1 \mathrm{~mW}$ resulting in a transmission range of 10 meters. However, for a typical path exponent of $\alpha=3$, the received signal at a node within 90 meters of the transmitter is likely to be above the receiver noise floor.

Moreover, it was observed in the relay channel [12] that utilizing unreliable overheard information was essential to achieving capacity. We borrow this idea and re-examine the minimum energy broadcast problem under the assumption that nodes exploit the energy of an unreliable reception. This idea is in particular suitable for the broadcast problem, where a node has multiple opportunities to receive a message as it is forwarded through the network. We refer to this cooperative strategy as accumulative broadcast.

Even in the simplest case of a single relay node, finding the maximum achievable common rate for a given set of transmit powers is, in general, an unsolved open problem. Even in the special case of the physically degraded relay channel, key techniques employed in [12] to enable coordination of the transmissions of the source and the relay in order to achieve capacity are not easily extensible to multiple node networks. In this work, we seek to employ overheard information in a large scale network. We focus on techniques that can be implemented as distributed network layer algorithms in which nodes use local information and coarse timing and synchronization. In particular, we make the following assumptions:

- Loose Synchronization: Nodes cannot synchronize transmissions for coherent signal combining at a receiver.

- Reliable Forwarding: A node can forward a message only after reliably decoding that message.

The advantages of coherent signaling and unreliable forwarding have been recognized for networks in which one or more relay nodes forward to a destination node, [12]-[15]. 
However, it is not apparent that coherent signal combining can be achieved simultaneously at multiple receivers nor is it clear that networks can support the precise sychronization of transmitting nodes and exact knowledge of radio path delays needed for coherent combining at a single receiver. By contrast, unreliable forwarding is practically implementable and has been shown to be superior to reliable forwarding in certain scenarios [15]. Nevertheless, we will see that reliable forwarding can simplify both the system architecture and the optimization of retransmission strategies, while still allowing us to benefit from unreliable overheard information.

Because it allows for more radiated broadcast energy to be captured, accumulative broadcast will increase the energy efficiency of broadcasting in any wireless network. However, the focus of our work will be on networks operating in the wideband regime [16] where the spectral efficiency is low. This assumption was motivated by applications for wireless sensor networks, where power, rather than bandwidth, is the limiting resource. Thus, the data rate is very small compared to the bandwidth, resulting in a low spectral efficiency. In the sensor networks where the energy-efficiency is the primary goal [17], operating in the wideband regime seems like the right choice: at the expense of using the large number of degrees of freedom per transmitted bit, the transmit energy per bit can be minimized [18]. However, finding the minimum energy per bit in networks with relays is still an open problem.

We will show that for a network operating in the wideband regime, the forwarding nodes can employ a simple repetition coding strategy in which all the nodes use the same codebook. While there is a benefit from using more general codes with incremental redundancy [19] in a general wireless network, this benefit diminishes when broadcasting in a network operating in the wideband regime [20].

The assumption of large bandwidth resources allows for transmission of different nodes to occur in orthogonal channels. The maximum achievable rate in a one-relay channel is then known [21]. For the network, orthogonal signaling enables us to determine the maximum achievable rate using repetition coding strategy, at every node, and to formulate the accumulative broadcast problem. Since during the accumulative broadcast, more radiated energy is captured than by using the minimum-energy broadcast tree approach, it is straightforward to show [22] that accumulative broadcast results in a more energy-efficient solution.

As we will show, finding the best solution to the accumulative broadcast problem is NP-complete. This motivates an efficient heuristic algorithm. Initially, we propose a centralized algorithm that requires global knowledge of the channel gains. However, centralized algorithms are not well suited for sensor networks consisting of many nodes which are all limited in power and computational resources [23]. For the minimum-energy broadcast problem, localized distributed algorithms were proposed in [5] and [6]. Both solutions rely on a distributed algorithm for constructing minimum-weight spanning trees in undirected and directed graphs, [24], [25]. Other localized algorithms for broadcasting were suggested recently in [26] and [10]. Two distributed versions of BIP were presented in [27]. In this paper, we present a distributed version of the accumulative broadcast heuristic algorithm that uses only local information at the nodes.

This paper is organized as follows. In the following section, we give the network model. In Section III, we formulate the Accumulative Broadcast problem and show that the problem is NP-complete. A centralized greedy filling heuristic and its performance are presented in Section IV. A distributed version of the greedy filling algorithm is given in Section V. The proofs for the theorems are given in the Appendix.

\section{SYSTEM MODEL.}

We consider a stationary wireless network of $N$ nodes such that from each transmitting node $k$ to each receiving node $m$, there exists an AWGN channel of bandwidth $W$ characterized by a frequency non-selective link gain $h_{m k}$. In our analysis, we do not consider fading and thus each channel is time-invariant with a constant link gain representing the signal path loss. We further assume sufficient bandwidth resources to enable each transmission to occur in an orthogonal channel, thus causing no interference to other transmissions. Each node has both transmitter and receiver capable of operating over all channels.

A receiver node $j$ is said to be in the transmission range of transmitter $i$ if the received power at $j$ is above a threshold that ensures the capacity of the channel from $i$ to $j$ is above the code rate of node $i$. We assume that each node can specify its power level, which will determine its nominal transmission range. Nodes beyond this transmission range will receive an unreliable copy of the transmitted signal. These nodes can exploit the fact that a message is sent through multiple hops on its way to all the nodes since repeated transmissions act as a repetition code for all nodes beyond the transmission range.

We view each orthogonal channel as a discrete-time Gaussian channel by representing a waveform of duration $T$ as a vector in the $n=2 W T$ dimensional space [28]. Then, during the $i$ th slot, a source node, labeled node 1 , transmits a codeword (vector) $X^{n}(i)$ from a $\left(2^{n R}, n\right)$ Gaussian code that is generated according to the distribution $p\left(X^{n}\right)=\prod_{l=1}^{n} p\left(x_{l}\right)$ where $p(x) \sim N(0,1)$. Under the reliable forwarding constraint, a node $j$ is permitted to retransmit (forward) codeword $X^{n}(i)$ only after reliably decoding $X^{n}(i)$. With an appropriate set of retransmissions, eventually every node will have reliably decoded $X^{n}(i)$. Henceforth, we drop the index $i$ and focus on the broadcast of a single codeword $X^{n}$. We will say a node is reliable once it has reliably decoded $X^{n}$.

The constraint of reliable forwarding imposes an ordering on the network nodes. In particular, a node $m$ will decode $X^{n}$ from the transmissions of a specific set of transmitting nodes that became reliable prior to node $m$. Starting with node 1 , the source, as the first reliable node, a solution to the accumulative broadcast problem will be characterized by a reliability schedule, which specifies the order in which the nodes become reliable. Given a reliability schedule, we can determine the maximum achievable rate at every node.

A reliability schedule $\left[n_{1}, n_{2}, n_{2}, \ldots, n_{N}\right]$ is simply a permutation of $[1,2, \ldots, N]$ that always starts with the source node $n_{1}=1$. Given a reliability schedule, it will be convenient to relabel the nodes such that the schedule is simply 
$[1,2, \ldots, N]$. After each node $k \in\{1, \ldots, m-1\}$ transmits codeword $X^{n}$ with average energy per symbol $P_{k}$, the received signal at node $m$ for each symbol $x$ in the codeword is

$$
\mathbf{y}_{m}=\mathbf{h}_{m} x+\mathbf{n},
$$

where $\mathbf{h}_{m}=\left[\sqrt{h_{m 1} P_{1}}, \ldots, \sqrt{h_{m m-1} P_{m-1}}\right]^{T}$ has $k$ th element $\sqrt{h_{m k} P_{k}}$ equal to the received energy corresponding to the transmission of node $k$ and $\mathbf{n}$ is a random noise vector with covariance matrix $\mathbf{K}_{\mathbf{n}}=\sigma^{2} \mathbf{I}_{K}$. The mutual information is given by

$$
I\left(x ; \mathbf{y}_{m}\right)=\frac{1}{2} \log _{2}\left(1+\frac{\sum_{k=1}^{m-1} h_{m k} P_{k}}{\sigma^{2}}\right)
$$

as in a multi-antenna system with $m-1$ transmitting antennas and one receiving antenna [21]. It follows from (2) that the maximal number of bits per second that can be transmitted in the system given by (1) is

$$
r_{m}=W \log _{2}\left(1+\frac{\sum_{k=1}^{m-1} h_{m k} p_{k}}{N_{0} W}\right) \quad \text { bits/s, }
$$

where $p_{k}$ is the transmit power at node $k$ and $N_{0}$ is the onesided power spectral density of the noise.

Let the required data rate for broadcasting $\bar{r}$ be given by

$$
\bar{r}=W \log _{2}\left(1+\frac{\bar{P}}{N_{0} W}\right) \quad \text { bits/s. }
$$

Rate $\bar{r}$ has to be achieved at every reliable node $m$. From (3) and (4), achieving $r_{m}=\bar{r}$ implies that the total received power at node $m$ is above the threshold $\bar{P}$; that is, $\sum_{k=1}^{m-1} h_{m k} p_{k} \geq$ $\bar{P}$.

In the system where the nodes are power limited and the data rate $\bar{r}$ is small relative to the channel bandwidth $W$, the spectral efficiency $(\mathrm{b} / \mathrm{s} / \mathrm{Hz})$ is low and the system operates in the wideband regime [16]. The increase in rate with power is linear:

$$
\bar{r}_{\infty}=\lim _{W \rightarrow \infty} \bar{r}=\frac{\bar{P}}{N_{0} \log 2} \quad \text { bits/s. }
$$

We emphasize that the system operates at a low spectral efficiency due to the low transmit powers at the nodes and does not imply the large operating bandwidth $W$. From Equation (5), when communicating at rate $\bar{r}_{\infty}$, the required signal energy per bit has the minimum value $E_{b}=\bar{P} / \bar{r}_{\infty}=$ $N_{0} \log 2$ Joules/bit. Thus, the system uses the energy in the most economical way possible to communicate reliably [18] because the system uses a large number of degrees of freedom per information bit. This energy can be collected at a node $m$ during one transmission interval $[0, T]$ when a transmitter $j$ is signaling with power $p_{j}=\left(N_{0} \log 2\right) /\left(h_{m j} T\right)$. However, during the accumulative broadcast in the system (1), the required energy $E_{b}$ is collected in $m-1$ repeated transmissions. In the wideband regime, the maximum achievable rate at node $m$, as given by (3), becomes

$$
\lim _{W \rightarrow \infty} r_{m}=\frac{1}{N_{0} \log 2} \sum_{k=1}^{m-1} h_{m k} p_{k} .
$$

In [29], it was shown that TDMA is first-order optimal in the wideband regime as it achieves the minimum energy per information bit of a multiaccess channel. Using (6), it is straightforward to conclude that the first-order optimality is preserved even if the repetition code described above is employed. We formally state this conclusion in the next Theorem.

Theorem 1: For the wideband regime, with fixed transmitted powers $\left\{p_{1}, \ldots, p_{N}\right\}$ and a reliability schedule $[1,2, \ldots, N]$, the maximum rate achievable from the source to node $m$ is given by Equation (6) and is achieved by repetition coding.

\section{APPROACH}

Under the constraint of reliable forwarding, an optimal solution to the minimum energy accumulative broadcast problem must specify the reliability schedule as well as the transmitter power levels used at each node. An optimal choice of the reliability schedule will result in minimum total transmitted power over the set of nodes. The problem has some similarity to the minimum-energy broadcast problem [2], [4]-[7], [9] in that the optimum solution involves the right ordering of relay nodes and transmit power levels. In the minimum-energy broadcast problem, the broadcast tree uniquely determines the transmission levels and thus solves the problem completely; a relay that is the parent of a group of siblings in the broadcast tree transmits with the power needed to reliably reach the most disadvantaged sibling in the group. Hence, the arcs in the broadcast tree uniquely determine the power levels for each transmission. In accumulative broadcast, however, there is no a clear parent-child relationship between nodes because nodes collect energy from the transmissions of many nodes. Furthermore, the optimum solution may require that a relay transmits with a power level different from the level precisely needed to reach a group of nodes reliably; the nodes may collect the rest of the needed energy from the future transmissions of other nodes. In fact, the optimum solution often favors such situations because all nodes beyond the range of a certain transmission are collecting energy while they are unreliable; the more such nodes, the more efficiently the transmitted energy is being used.

The differences between accumulative broadcast and the minimum-energy broadcast tree dictate a new approach. The crucial step is finding the best reliability schedule. Given a schedule, we can formulate a linear program (LP) that will find the optimum solution for that schedule. Such a solution will identify those nodes that should transmit and their transmission power levels. Solving the LP for all possible schedules and taking the minimum-energy solution among all the LP solutions will result in the optimum schedule, and optimum transmission power levels. This divides the problem into two subproblems.

To define the LP for a certain schedule, we use the observation that every node selected to transmit by the optimum solution, needs to transmit only once. This fact is given by the next theorem.

Theorem 2: In the wideband regime, given a solution to the accumulative broadcast problem consisting of a sequence of transmissions where a node $j$ is assigned to transmit $K$ times 
with power levels $P_{j}^{1}, \ldots P_{j}^{K}$, there is a feasible optimum solution in which node $j$ transmits once with power level $\sum_{k=1}^{K} P_{j}^{k}$.

A reliability schedule can be represented by a matrix $\mathbf{X}$ where

$x_{i j}= \begin{cases}1 & \text { if node } i \text { is scheduled to transmit after node } j \\ 0 & \text { otherwise }\end{cases}$

Each $x_{i j}$ is an indicator that a node $i$ collects energy from a transmission by node $j$. Note that $x_{i i}=0$, for all $i$ and $x_{j i}=1-x_{i j}$. Given a schedule $\mathbf{X}$, we define a gain matrix $\mathbf{H}(\mathbf{X})$ with element $(i, j)$ given by $h_{i j} x_{i j}$. In terms of the vector $\mathbf{p}$ of transmitted powers, the LP for schedule $\mathbf{X}$ is

$$
\begin{aligned}
\rho(\mathbf{X})=\min \mathbf{1}^{T} \mathbf{p} \\
\text { subject to } \mathbf{H}(\mathbf{X}) \mathbf{p} \geq \mathbf{1} \bar{P}, \\
\mathbf{p} \geq \mathbf{0} .
\end{aligned}
$$

The inequality $\mathbf{H}(\mathbf{X}) \mathbf{p} \geq \mathbf{1} \bar{P}$ contains $N-1$ constraints requiring that the received power at all the nodes but the source is above the required threshold $\bar{P}$. Given a schedule $\mathbf{X}$, we will use $\mathbf{p}^{*}(\mathbf{X})$ to denote a power vector $\mathbf{p}$ that achieves total transmitted power $\rho(\mathbf{X})$.

In a schedule, all $N$ nodes are given a chance to transmit since $p_{j}$ can be greater than 0 for every node. Since the source always transmits first, there are $(N-1)$ ! schedules corresponding to the number of permutations of $N-1$ elements. Thus, out of $N^{(N-2)}$ broadcast trees, we consider a subset of $(N-1)$ ! schedules. If the best solution is that only a subset of nodes should be transmitting, the LP for the best schedule will find that solution by setting appropriate powers to zero. In general, however, the problem of finding a best schedule is intractable.

Theorem 3: The existence of a schedule $\mathbf{X}$ such that $\rho(\mathbf{X}) \leq B$ is an NP-complete problem.

\section{SCHEDUling HeURistic}

Because of the intractability of finding the best schedule, we now propose a heuristic algorithm that finds a good schedule. Once the schedule is determined, the LP for that schedule is solved to find the optimum power levels. We evaluate the performance of the algorithm through simulation and compare its power efficiency to the optimum solution as well as to the performance of BIP.

We observe that we can restrict ourselves to scheduling nodes in an order in which they can become reliable one at a time. When a node $j$ is scheduled to be the next node in a schedule after a set of nodes $S$, then a transmission from that set has to make node $j$ reliable. If the power that is needed to reach node $j$ is enough to reach another unreliable node $i$ as well, then we could have done better by assigning node $i$ for transmission before node $j$. This is because $i$ cannot benefit from a transmission from node $j$ (since it is made reliable before $j$ ) but $j$ might benefit from a transmission from $i$. If, in fact the optimal solution is to simultaneously make the two nodes $i$ and $j$ reliable by a transmission from the same set $S$, then those two nodes do not need to overhear each other's transmission. Thus, all the schedules in which nodes $i$ and $j$ are scheduled one right after the other in any order, will

$$
\begin{aligned}
& \mathbf{s}=[1] ; \quad \mathbf{p}=0 \\
& \text { while }(|S|<N) \text { do } \\
& \qquad \begin{aligned}
k= & \arg \max _{i \in S} \sum_{j \in U} h_{j i} ; \\
j= & \arg \min _{m \in U}\left(\bar{P}-\sum_{i \in S} h_{m i} p_{i}\right) / h_{m k} ; \\
& p_{k} \leftarrow p_{k}+\left(\bar{P}-\sum_{i \in S} h_{j i} p_{i}\right) / h_{j k} ; \\
& \mathbf{s} \leftarrow[\mathbf{s}, j]
\end{aligned}
\end{aligned}
$$

end

Given a partial schedule s, $S$ is the unordered set of nodes in $\mathbf{s}$ and its complement $U$ is the set of unreliable nodes. The cardinality of $S$ is given by $|S|$.

Fig. 1. Greedy Filling Algorithm.

have the same performance. This reasoning will be used in the proposed greedy filling heuristic algorithm.

The algorithm pseudocode is given in Figure 1. The algorithm starts with a partial reliability schedule $\mathbf{s}=[1]$ that contains only the source. Given a partial schedule s, a step of the greedy filling algorithm does the following:

1) We find the reliable node $k$ that maximizes the fill rate of the unreliable set $U$, where the fill rate,

$$
R_{k}=\sum_{j \in U} h_{j k}
$$

is the sum of the link gains from node $k$ to the set $U$ of all unreliable nodes.

2) We increase $p_{k}$ such that the transmission by $k$ adds one more node, node $j$, to the reliable set.

3) We append node $j$ to the partial schedule.

Once the schedule is complete, the LP is solved to find the optimum power levels for that schedule.

We evaluated the performance of the algorithm and compared it to the optimal solution as well as to the performance of BIP for networks with a small number $(5-10)$ of randomly positioned nodes. We also compared the performance of two heuristics for more dense networks with a maximum of 150 nodes. Nodes were uniformly distributed in an area of size $10 \times 10$. The transmitted power was attenuated as $d_{j k}^{\alpha}$ for three different values of propagation exponent $\alpha=2,3,4$. The received power threshold was chosen to be $\bar{P}=1$. Results were based on the performance of 100 randomly chosen networks. In small networks, the performance metric used was the normalized total transmit power in the network. In each simulation run, the power used when a heuristic algorithm was employed was normalized by the power used in the optimum solution. Results are shown in Figure 2 as a function of the number of network nodes for $\alpha=2$. Results show the heuristic algorithm performance very close to the optimum. This is a desirable and important characteristic, given the complexity of finding the optimum solution. Simulation results also show a noticeable $1.7 \mathrm{~dB}$ savings in average power of accumulative broadcast over the minimum-energy broadcast tree found by BIP.

For networks with a larger number of nodes, performance comparison of the greedy filling algorithm and BIP are shown in Figure 3. The metric used was the average total power used 


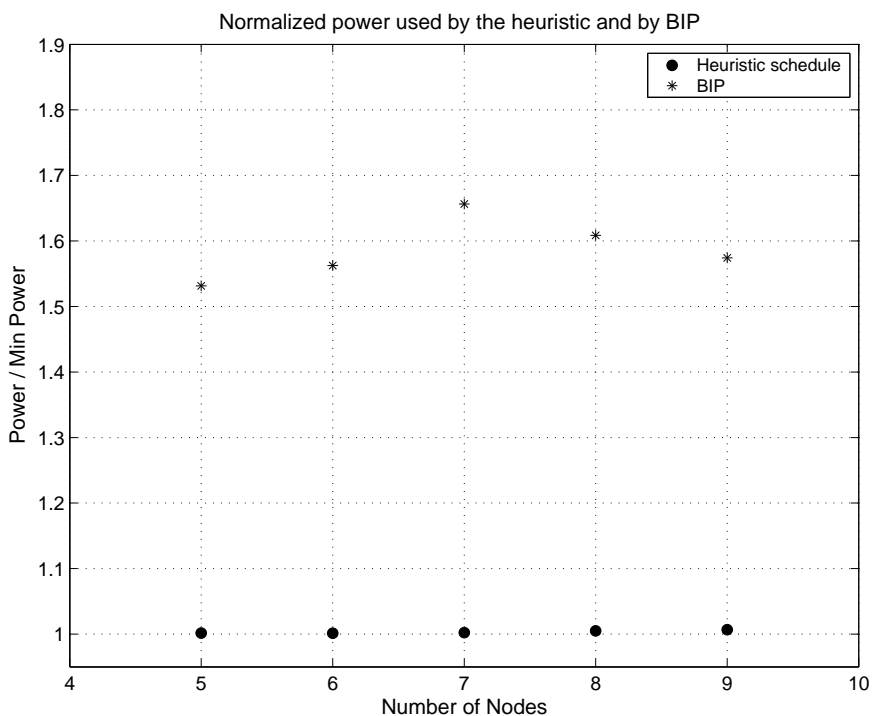

Fig. 2. Normalized power used for broadcasting.

for broadcasting. We observe that total power decreases with the number of nodes due to the increased number of shorter hops. The decrease in the case of the accumulative broadcast is steeper since the increased number of transmissions allows for more energy to be collected. Hence the relative improvement over BIP increases with the node density of the network. For smaller values of propagation exponent $\alpha$, the smaller path loss allows for the higher gains from the accumulative broadcast and we observe up to $5 \mathrm{~dB}$ savings per node for $\alpha=2$. Results also show that, for a larger number of nodes the total power required is smaller for larger values of $\alpha$. This counterintuitive result occurs in dense networks when most distances $d_{j k}$ become less than 1 so that $1 / d_{j k}^{4}>1 / d_{j k}^{2}$

Figure 3 also shows the loss in the performance when the LP is not employed to determine the optimum power levels for a greedy filling schedule. Instead, the power levels $\left\{p_{k} \mid k=1, \ldots N\right\}$ found by the greedy filling algorithm are used for broadcasting. We observe only a small loss in the performance. Thus, finding the optimum power levels is not as crucial as finding a good schedule. We will use this observation to formulate the distributed version of the greedy filling algorithm next.

\section{Distributed Algorithm}

In the greedy filling algorithm, we assumed full knowledge of the link gains when forming a schedule. In particular, we assumed that the fill rates of the reliable nodes are known in every step, so that the transmitting node $k$ could be chosen. Also, we assumed that the transmit power needed to make one more node reliable could be determined. In this section, we propose a distributed version of the greedy filling algorithm that assumes only local information at the nodes. The distributed algorithm is based on the observation that the greatest contribution to a fill rate of a reliable node $i$ will be made by the link gains to its neighbors that, together with node $i$, define a neighborhood $N_{R}(i)$ of node $i$. As we specify later,

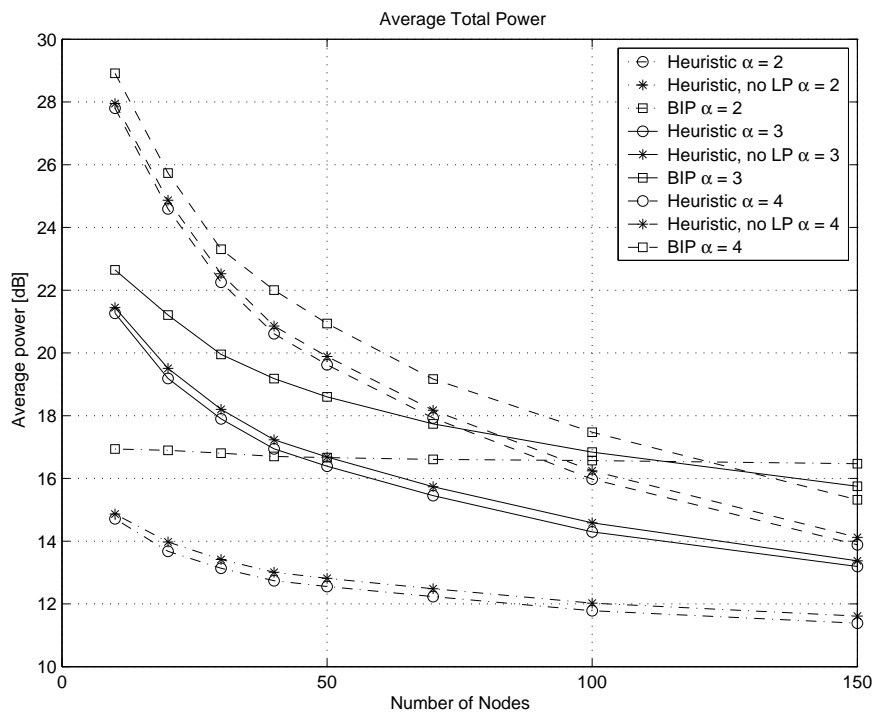

Fig. 3. Average total power used for broadcasting.

the transmit broadcast energy of node $i$ will be determined by acknowledgments (ACKs) sent by unreliable neighbors as they become reliable. In addition, ACK control packets will allow node $i$ to determine the neighborhood $N_{R}(i)$. Specifically, any ACK packet will be sent with a fixed power level $P_{\mathrm{c}}$ and rate $r_{c}$ chosen to guarantee the network connectivity [30]. Distributed algorithms for determining such a power level have been proposed (see [31], [32]). The neighborhood $N_{R}(i)$ is then defined as the set of nodes that receive the ACK from node $i\left(\mathrm{ACK}_{i}\right)$, with received power above a threshold $\bar{P}_{N_{R}}$ that assures reliable reception. Neighborhood $N_{R}(i)$ thus contains node $i$ and all nodes that are within some range $R$ from node $i$. The formed links are bidirectional which is desirable in a wireless network [10], [33]. All control packets used in the algorithm will be sent reliably within each neighborhood.

We assume that packet headers are short in comparison to long code words so that the energy used to transmit a header is negligible. Thus we assume that the physical header is always received reliably, even for packets received unreliably. This allows for both symbol-level and packet-level synchronization. It also allows a node to distinguish between data and control packets and determine the packet sequence numbers as well transmitter IDs. Because each node will operate in a different channel, a node identity can also be determined by the channel used.

We now give a detailed description of the distributed algorithm. The algorithm pseudocode is given in Figure 4. We let $S_{i}=S \cap N_{R}(i)$ and $U_{i}=U \cap N_{R}(i)$ denote respectively reliable and unreliable neighbors of node $i$. Initially, each node $i$ sets $S_{i}=\emptyset$. While node $i$ is unreliable, node $i$ will collect the energy of overheard transmissions including those from nodes outside its neighborhood. In addition, it will listen for any $\mathrm{ACK}_{j}$ that is received with power above the threshold $\bar{P}_{N_{R}}$. When it receives $\mathrm{ACK}_{j}$, node $i$ will identify that $j \in N_{R}(i)$ and will respond by sending a link gain $(L G)$ control packet containing $h_{i j}$ reliably to node $j$. This also informs node $j$ 
that $i \in N_{R}(j)$. Node $i$ will then update $S_{i}$ by adding node $j$ to $S_{i}$.

Once it becomes reliable, node $i$ will itself send an $\mathrm{ACK}_{i}$. This $\mathrm{ACK}_{i}$ will prompt every node $j$ in $U_{i}$ to send a $L G$ packet to node $i$, enabling node $i$ to calculate its fill rate $R_{i}$ and broadcast it in $N_{R}(i)$ using a control packet $\mathrm{FR}_{i}$.

After a reliable node $i$ receives an $\mathrm{ACK}_{j}$, it will do the following: if it was transmitting data at the time it received an $\mathrm{ACK}_{j}$, it will stop transmitting. It will update sets $S_{i}$ and $U_{i}$ by moving node $j$ from $U_{i}$ to $S_{i}$, update its fill rate $R_{i}$ and notify its neighbors of its new fill rate. The reliable node that has the maximum fill rate in $S_{i}$ will then transmit.

At all times prior to its first transmission, node $i$ will keep track of the identity of the reliable neighbor (a node in $S_{i}$ ) from which it last received the data packet. Thus, in case node $i$ decides to transmit next, it will know the node whose transmission preceeded its own and will use that information for future data broadcasting. The algorithm will stop at a node $i$ when $U_{i}$ is empty.

When transmitting, node $i$ will repeatedly send the same data packet of duration $T$ at the rate $\bar{r}_{\infty}$ given by (5) and at the fixed low power level $p$ until it hears an $A C K$ after some time $T^{\prime}$. Each of these data packets will contribute $h_{j i} p T$ to the energy collected at the unreliable node $j$. Once the total collected energy at node $E_{j}$ becomes $E_{j}=\bar{P} T$, node $j$ will send an $\mathrm{ACK}_{j}$. By that time, node $i$ has transmitted $T^{\prime} / T$ data packets, it can determine that the actual transmit power level needed to make $j$ reliable was $p T^{\prime} / T$. Power level $p$ is assumed to be chosen small enough so that the negligible excessive power is received at an unreliable node before it has a chance to transmit an ACK. At the end of the algorithm, a node $i$ will know the total broadcast energy it used $E_{i}^{t}$, and thus the power level $P_{i}=E_{i}^{t} / T$ that it will use for time $T$, to transmit new data that will arrive. Node $i$ will also know the identity of the neighbor whose transmission it should follow. We assume that $P_{i}$ is small enough to allow the network to operate in the wideband regime.

In the algorithm, the action of nodes are triggered by receptions of the ACK messages and we let each step of the algorithm start with the transmission of an ACK. The algorithm will terminate in $N-1$ steps. It is easy to see that deadlocks cannot occur: Since the network is connected, every unreliable node will eventually have a reliable neighbor, causing the fill rate of that neighbor to be nonzero. At each step, one of the fill rates must be the maximum and therefore, at least one reliable node will decide to transmit. Once all the nodes are reliable, all the fill rates will be zero and the algorithm will stop.

The benefit of the overheard information will be highest in the neighborhood of a transmitting node. For that reason, the distributed greedy heuristic, unlike its centralized counterpart, allows simultaneous transmissions from nodes that are not in the same neighborhood.

We examine the impact of the limited knowledge at the nodes to the performance of the heuristic. Performance of the algorithm depends on the choice of range $R$. For large enough $R$, the performance of the distributed algorithm approaches the performance of its centralized version. For the smallest

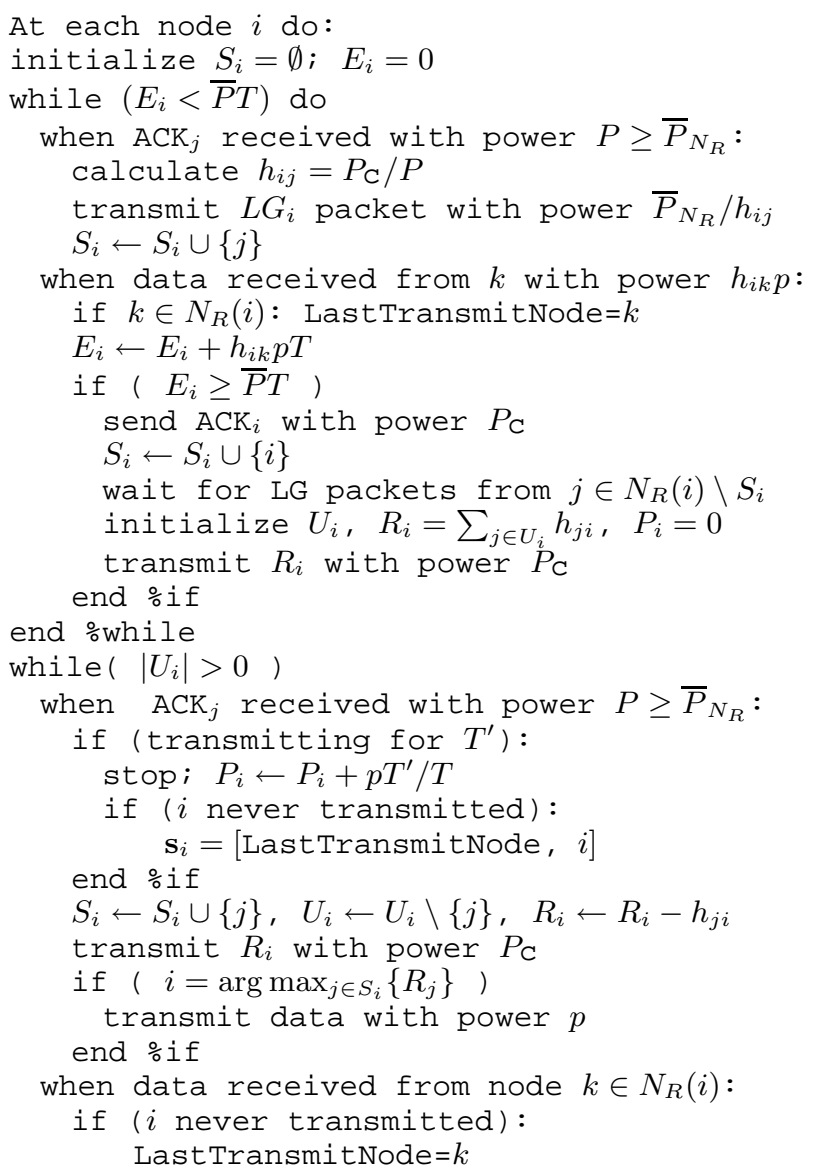
$\bar{P}_{N_{R}}$ is the received power threshold required in a neighborhood. For a node $i, E_{i}$ denotes energy collected from data packets and $R_{i}$ denotes its fill rate.

Fig. 4. Distributed Greedy Filling Algorithm.

$R$ that provided the network connectivity, Figure 5 shows the performance comparison of the distributed algorithm with its centralized counterpart as a function of node density. In this case, the actual power levels found by the greedy filling algorithm were used instead of the optimum power levels. Comparison with the centralized algorithm using an LP as well as with BIP, is shown in Figure 6 . We observe that the performance of the distributed algorithm is close to the performance of its centralized version.

\section{CONCLUSION}

In this paper, we address the minimum-energy broadcast problem. To increase the energy efficiency, we propose accumulative broadcast which allows nodes outside of the transmission range to collect the energy of the unreliably received signal. Nodes will have multiple opportunities to reliably receive the message as the message is forwarded through the network. This approach allows for more radiated broadcast energy to be captured at the nodes and hence improves the energy efficiency of the broadcast. We prove that finding the optimum schedule for this problem is NP-complete. It has come to our attention that under a different physical model 


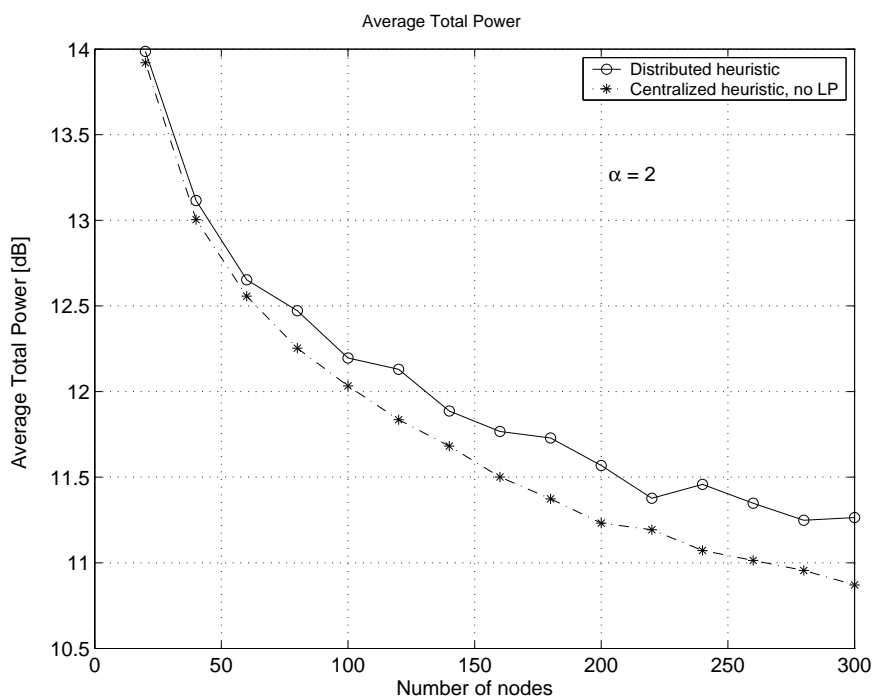

Fig. 5. Performance comparison between distributed and centralized versions of the algorithm.

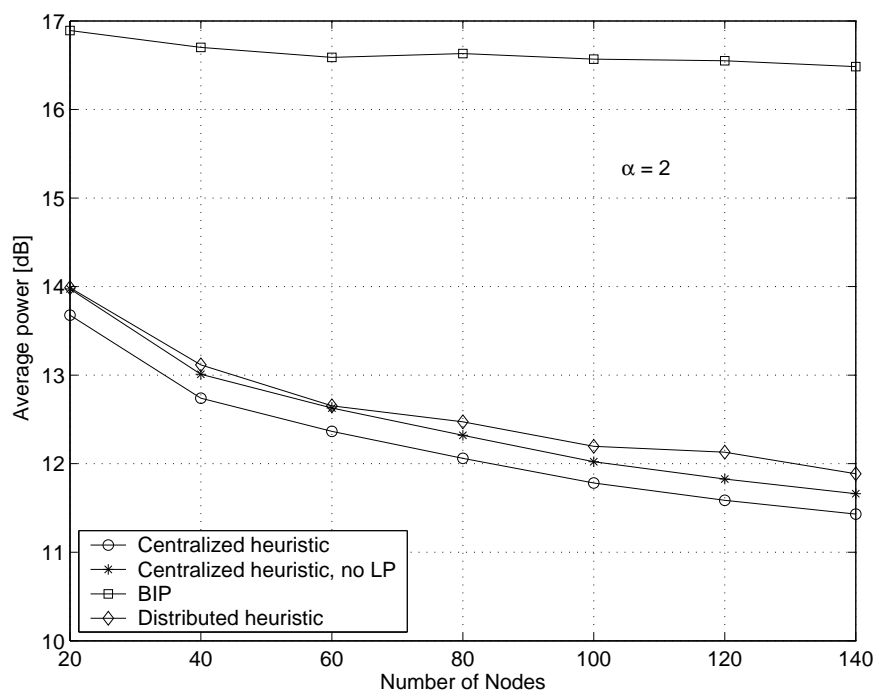

Fig. 6. Performance comparison between distributed and centralized versions of the algorithm as well as BIP.

for cooperative broadcast, this same result was independently derived in [34].

We propose a heuristic algorithm that finds energy-efficient solutions and can still provide energy savings compared to the minimum-energy broadcast tree approach. We then present a distributed version of the algorithm that uses only local knowledge at the nodes which is better suited for application in networks consisting of a large number of power-limited nodes.

Our preliminary results suggest that accumulative broadcast merits further study. In particular, it would be interesting to consider the implications of time varying channels. Even in the case of the ergodic flat fading channel, variety of different problems arise depending on the availability of channel state information (CSI) at the transmitter and/or receiver.

If CSI is known at a receiving node only, rate (2), averaged over all the fading realizations is still achievable, provided that the codewords are long enough to span all the channel states [21]. Although TDMA can no more achieve the maximum sum rate, it can still achieve the minimum energy per bit and thus it stays first-order optimal in the wideband regime, even in the presence of fading [29]. Because transmitting nodes have no CSI, the proposed algorithms for accumulative broadcast would have to be based on the link gain statistics (averages).

Knowledge of the CSI at the transmitters may give an opportunity to exploit the channel variations through power control. This assumption would demand a re-examination of the Section II system model since it is not immediately clear if the same scheme of using the same codebook, and thus the same code rate, at all nodes is appropriate. During the accumulative broadcast, there is a multiple access channel between all the reliable nodes to any unreliable node. In such a multiuser setting, it was shown that the optimal power control can significantly increase the capacity [35]. The increase in capacity is achieved by the random TDMA approach in which a user with the best fading conditions transmits. Since for a large number of users there is likely to be a user with a good channel, such an approach benefits from the diversity gain. Because it is inherent in a wireless network with many users, this gain is refered to as multiuser diversity and can also be exploited when the channel variations are due to the mobility [36]. In accumulative broadcast, this would mean that a reliable node should transmit when there is an unreliable node in its approximity, in the manner of Infostations [37]. In our scenario, an additional dimension arises because there are multiple receivers (unreliable nodes) for almost every transmission. Its impact on the optimal choice of a transmitter and the power allocation is to be determined.

Furthermore, multiuser problem [35] extended nicely to the case of frequency-selective channel, allowing for the flat fading solution to be applied in each frequency subband [38]. For a network operating in the wideband regime, the extension to the frequency-selective channel model is appropriate and the accumulative broadcast problem in the frequency-selective channel is still to be addressed.

\section{Appendix: Additional Proofs}

Proof (Theorem 1): An upper bound to the achievable rate between the source and the destination is the maximum conditional mutual information across a minimum cut [28]. Consider the multiaccess cut in the given network that separates the destination node from the rest of the network. Let $X_{j}$ denote a symbol transmitted at node $j$ and $Y$ denote the received signal at the destination. The maximum mutual information across this cut is given by

$$
C_{\mathrm{MAC}}=I\left(X_{1}, \ldots X_{m-1} ; Y\right) .
$$

In this network, each orthogonal channel is assigned bandwidth $W$ and hence the mutual information above is given by the sum of rates achieved in each of the channels. For Gaussian channels,

$$
C_{\mathrm{MAC}}=W \sum_{k=1}^{m-1} \log _{2}\left(1+\frac{h_{m k} p_{k}}{N_{0} W}\right) .
$$


In the wideband regime, Equation (11) becomes

$$
\begin{aligned}
C_{\text {MAC }} & =\lim _{W \rightarrow \infty} W \sum_{k=1}^{m-1} \log _{2}\left(1+\frac{h_{m k} p_{k}}{N_{0} W}\right) \\
& =\frac{1}{N_{0} \log 2} \sum_{k=1}^{m-1} h_{m k} p_{k},
\end{aligned}
$$

which is precisely the rate given by (6) achieved using the repetition strategy. Since this rate is achievable, this cut is the minimum cut. No better rate can be achieved since it would violate the condition for the upper bound.

Proof (Theorem 2): For the purpose of this proof we represent a solution to the accumulative broadcast problem by a vector with each entry $i$ containing the $i$ th transmitting node $n_{i}$ and the $i$ th transmitted power level $P_{i}$. A solution $\mathbf{S}$ is represented as

$$
\mathbf{S}=\left[\begin{array}{llll}
\left(n_{1}, P_{1}\right) & \left(n_{2}, P_{2}\right), & \ldots & \left(n_{M}, P_{M}\right)
\end{array}\right]^{T}
$$

for some $M \geq N$. We write $\left(n_{i}, P_{i}\right)=(0,0)$ if no node transmits at step $i$.

Assume that $\mathbf{S}$ schedules the same node for a transmission more than once. It is sufficient to show that there is a feasible schedule $\hat{\mathbf{S}}$ that uses the same total transmit power as $\mathbf{S}$, in which that node transmits once. Let $l$ denote the smallest integer such that there exists an integer $m>l$ with $n_{m}=n_{l}$. Consider the policy $\hat{\mathbf{S}}$, a vector of length $M-1$ with elements $\left(\hat{n}_{i}, \hat{P}_{i}\right)$ such that

$$
\left(\hat{n}_{i}, \hat{P}_{i}\right)= \begin{cases}\left(n_{l}, P_{l}+P_{m}\right) & \text { if } i=l \\ (0,0) & \text { if } i=m \\ \left(n_{i}, P_{i}\right) & \text { if } i \geq m\end{cases}
$$

The solution $\hat{\mathbf{S}}$ combines transmissions at steps $l$ and $m$ into a single transmission with power $P_{l}+P_{m}$ at step $l$. The rest of the nodes are scheduled as in $\mathbf{S}$.

For any node $j$, the energy accumulated by step $k$ in new schedule is $\sum_{i=1}^{k-1} h_{j \hat{n}_{i}} \hat{P}_{i} \geq \sum_{i=1}^{k-1} h_{j n_{i}} P_{i}$. Therefore, $\hat{\mathbf{S}}$ is a feasible schedule since any node $j$ made reliable by step $k$ in schedule $\mathbf{S}$ is also reliable at step $k$ in the new schedule. $\square$

Proof (Theorem 3): Let $\Pi_{i}$ denote the set of all vectors $\pi=\left[\pi_{0}, \ldots, \pi_{i}\right]$ that are permutations of $[0,1, \ldots, i]$. A formal statement of the ACCUMULATIVE BROADCAST (AB) problem is

AB Given a nonnegative matrix specified by
$\left\{h_{j, k} \mid 1 \leq j \leq m, 0 \leq k \leq m\right\}$ and a constant
$c$, does there exist a permutation $\pi \in \Pi_{k}$
with $\pi_{0}=0$ and a non-negative vector
$\mathbf{p}=\left[p_{0}, p_{1}, \ldots, p_{m}\right]$ such that $\sum_{k=0}^{m} p_{k} \leq c$
and $\sum_{k=0}^{j-1} h_{\pi_{j}, \pi_{k}} p_{\pi_{k}} \geq 1, \quad j=1, \ldots, m$.

Thus an instance of $\mathrm{AB}$ is specified by the pair $\left(\left\{h_{j, k}\right\}, c\right)$. Note that we set the reliability threshold to unit power since any scaling can be specified by the constant $c$. We observe that $\mathrm{AB}$ is in NP since given a permutation $\pi$ and vector $\mathbf{p}$, it is easy to check whether the $\mathrm{AB}$ constraints are met.

We will show that the ACCUMULATIVE BROADCAST problem is NP complete by a polynomial time reduction of the DIRECTED HAMILTON PATH (DHP) [3] problem. Formally the DHP problem is
DHP Given a directed graph $G=(V, A)$ with nodes $V=\{0, \ldots, n\}$, does there exist a permutation $\pi \in \Pi_{n}$ such that $\pi_{0}=0$ and $\left(\pi_{i}, \pi_{i+1}\right) \in A$ for $i=0, \ldots, n-1$.

We now describe the transformation of DHP into an instance of $\mathrm{AB}$. Without loss of generality, we assume that the instance of DHP is such that node 0 has a single outgoing arc $(0,1)$ and that node $n$ is a sink node reachable by an $\operatorname{arc}(i, n)$ from each node $i \in\{1, \ldots, n-1\}$. Note that if this condition does not hold, we can add such source and sink nodes and solve an equivalent DHP. Thus, for each such graph, the Hamilton path, if it exists, will start at node 0 and terminate at node $n$.

Given $G=(V, A)$ for DHP, we construct a set of nodes $G^{\prime}$ and matrix $\left\{h_{j, k}\right\}$ for an instance of AB. In particular, for each node $k \in G$, we construct a cluster of nodes $C_{k} \subset G^{\prime}$. In particular, the cluster $C_{k}$ includes a node $i_{j, k}$ for each incident $\operatorname{arc}(j, k) \in A$ and a node $o_{k, l}$ for each outgoing arc $(k, l) \in A$. That is, in terms of each arc $(j, k) \in A$, we have created an incident node $i_{j, k} \in C_{k}$ and an outgoing node $o_{j, k} \in C_{j}$. Note that cluster $C_{0}$ contains only the single node $o_{0,1}$ and that the sink node $n$ has the cluster $C_{n}=\left\{i_{j, n} \mid 1 \leq j<n\right\}$ of only incoming nodes.

To avoid an explicit enumeration of the nodes in $G^{\prime}$, we describe the matrix $\left\{h_{j, k}\right\}$ in terms of a function $h(a, b)$ that gives the channel gain from node $b$ to node $a$. Similarly, we will use the notation $p(a)$ to denote the transmitted power of the node $a$. Corresponding to each arc $(j, k) \in A$, we have $h\left(i_{j, k}, o_{j, k}\right)=1$. Within each cluster $C_{k}$, we have that for any pair of incident nodes $i_{j, k}$ and $i_{j^{\prime}, k}, h\left(i_{j, k}, i_{j^{\prime}, k}\right)=1$. In addition, for each outgoing node $o_{k, l} \in C_{k}$, and each incoming node $i_{j, k} \in C_{k}, h\left(o_{k, l}, i_{j, k}\right)=1$. For all other pairs of nodes $a, b \in G^{\prime}$, we set $h(a, b)=0$. Keep in mind that if $h(a, b)=1$, then $p(b)=1$ yields received power $h(a, b) p(b)=1$ at node $a$. We will see in our AB construction, each node $a$ will use power $p(a) \in\{0,1\}$.

To prove that $\mathrm{AB}$ is NP-complete, we show that the graph $G$ has a Hamilton path if and only if the resulting instance $(h(\cdot, \cdot), c=2 n)$ of $\mathrm{AB}$ is feasible. Consider a Hamilton path that starts at node zero and proceeds through all nodes to node $n$. Suppose the Hamilton path uses arc $(j, k)$, then for the $\mathrm{AB}$ problem, we set $p\left(o_{j, k}\right)=1, p\left(i_{j, k}\right)=1, p\left(o_{j, k^{\prime}}\right)=0$ for all $k^{\prime} \neq k$, and $p\left(i_{j^{\prime}, k}\right)=0$ for all $j^{\prime} \neq j$. In the context of $\mathrm{AB}$, node $o_{j, k}$ transmits to make node $i_{j, k}$ reliable and then node $i_{j, k}$ transmits to make all nodes in cluster $C_{k}$ reliable. If the next arc in the Hamilton path is $(k, l)$, then in the AB, $o_{k, l}$, which has already been made reliable by the transmission of $i_{j, k}$, will transmit to make $i_{k, l}$ reliable. We call the event that an incoming node $i_{j, k}$ is made reliable a visit to cluster $C_{k}$. The sequence of nodes in the Hamilton path corresponds exactly to the sequence of cluster visits. To calculate the total transmitted power, note that in cluster $C_{0}$, node $o_{0,1}$ will transmit. In clusters $1, \ldots, n-1$, one incoming node and one outgoing node will transmit. Lastly, in cluster $C_{n}$, one incoming node will transmit to make the other incoming nodes in $C_{n}$ reliable. The total transmitted power will be exactly $2 n$. We note that the node ordering required by the formal statement of $\mathrm{AB}$ will not be uniquely specified. If cluster $C_{k}$ is visited before cluster $C_{l}$, then all nodes in $C_{k}$ must be 
ordered ahead of nodes in $C_{l}$. In a cluster $C_{k}$, if incoming node $i_{j, k}$ is made reliable then $i_{j, k}$ must be first in the cluster but other nodes in the cluster can be ordered arbitrarily.

To complete the proof, suppose we have a solution to the $\mathrm{AB}$ problem. This $\mathrm{AB}$ solution must make every node in the graph $G^{\prime}$ reliable. For each cluster $C_{k}, 1 \leq k \leq n$, at least one incoming node $i_{j, k}$ must be made reliable by the transmission of the corresponding outgoing node $o_{j, k}$. However, since this transmission of $o_{j, k}$ makes only $i_{j, k}$ reliable, one such transmission is needed for each cluster $C_{k}$. Over all clusters $C_{k}, 1 \leq k \leq n$, we require $n$ such transmissions. Further, within each cluster, the outgoing nodes can be made reliable only by the transmission of an incoming node in the cluster. Thus for each cluster $C_{k}, 1 \leq k \leq n$, at least one incoming node $i_{j, k}$ must transmit to make all other nodes in the cluster reliable; this requires $n$ additional transmissions. Thus $2 n$ is a lower bound to the number of transmissions for the $\mathrm{AB}$ problem. Moreover, if the solution to $\mathrm{AB}$ achieves the minimum $2 n$, then each outgoing node transmission must be to an incoming node in a cluster that has had no other incoming nodes receive a transmission from its corresponding outgoing node. That is, each cluster can be visited only once for the $2 n$ lower bound to be met. Starting with node 0 and cluster $C_{0}$, node $o_{0,1}$ will transmit to make node $i_{0,1}$ reliable. Node $i_{0,1}$ must then transmit to make all other nodes in cluster $C_{1}$ reliable. An outgoing node $o_{1, k}$ will then transmit to make a node $i_{1, k}$ reliable, constituting a visit to cluster $k$. To achieve the $2 n$ lower bound, each cluster will be visited precisely once, with termination at cluster $C_{n}$. Since moving from cluster $C_{j}$ to visit $C_{k}$ can occur only if $(j, k)$ is an arc in $G$, the AB solution corresponds to a Hamilton path in the graph $G$.

\section{REFERENCES}

[1] B. Williams and T. Camp, "Comparison of broadcasting techniques for mobile ad hoc networks," in Proc. of International Symposium on Mobile Ad Hoc Networking and Computing (MobiHoc 2002), June 2002, pp. 194-205.

[2] J. Wieselthier, G. Nguyen, and A. Ephremides, "On the construction of energy-efficient broadcast and multicast trees in wireless networks," in Proc. of INFOCOM'O0, Mar. 2000.

[3] C. Papadimitriou and K. Steiglitz, Combinatorial Optimization: Algorithms and Complexity. Prentice Hall, Englewood Cliffs, NJ, 1982.

[4] F. Li and I. Nikolaidis, "On minimum-energy broadcasting in all-wireless networks," in Proc. of Local Computer Networks (LCN 2001), Nov. 2001.

[5] A. Ahluwalia, E. Modiano, and L. Shu, "On the complexity and distributed construction of energy-efficient broadcast trees in static ad hoc wireless networks," in Proc. of Conf. on Information Science and Systems, Mar. 2002.

[6] M. Cagalj, J. Hubaux, and C. Enz, "Energy-efficient broadcast in allwireless networks," ACM/Kluwer Mobile Networks and Applications (MONET); to appear, 2003.

[7] W. Liang, "Constructing minimum-energy broadcast trees in wireless ad hoc networks," in Proc. of International Symposium on Mobile Ad Hoc Networking and Computing (MobiHoc'O2), June 2002.

[8] D. Bertsekas and R.G.Gallager, Data Networks. Prentice Hall, Englewood Cliffs, NJ, 1992.

[9] P.-J. Wan, G. Calinescu, X.-Y. Li, and O. Frieder, "Minimum-energy broadcasting in static ad hoc wireless networks," Wireless Networks, vol. 8, pp. 607-617, 2002.

[10] N. Li and J. Hou, "BLMST: A scalable, power-efficient broadcast algorithm for wireless sensor networks," in 1st ACM Conference on Embedded Networked Sensor Systems (SenSys 2003); submitted, Apr. 2003.
[11] SIG, "Bluetooth specification version 1.0b," Tech. Spec., 1999.

[12] T. Cover and A. E. Gamal, "Capacity theorems for the relay channel," IEEE Trans. on Information Theory, vol. 25, no. 5, pp. 572-584, Sept. 1979.

[13] M. Gastpar and M. Vetterli, "On the capacity of wireless networks: The relay case," in Proc. of INFOCOM'02, June 2002.

[14] — - "On asymptotic capacity of gaussian relay networks," in Proc. of International Symposium on Information Theory (ISIT'02), 2002.

[15] J. N. Laneman, D. N. C. Tse, and G. W. Wornell, "Cooperative diversity in wireless networks: efficient protocols and outage behavior," IEEE Trans. on Information Theory, submitted.

[16] S. Verdú, "Spectral efficiency in the wideband regime," IEEE Trans. on Information Theory, vol. 48, no. 6, pp. 1319-1343, June 2002.

[17] K. Sohrabi, J. Gao, V. Ailawadhi, and G. Pottie, "Protocols for selforganization of a wireless sensor network," IEEE Personal Communications, pp. 16-27, Oct. 2000.

[18] S. Verdú, "On channel capacity per unit cost," IEEE Trans. on Information Theory, vol. 36, no. 5, pp. 1019-1030, Sept. 1990.

[19] G. Caire and D. Tuninetti, "The throughput of hybrid-ARQ protocols for the gaussian collision channels," IEEE Trans. on Information Theory, vol. 47, no. 5, pp. 1971-1988, July 2001.

[20] I. Maric and R. Yates, "Performance of repetition codes and punctured codes for accumulative broadcast," in Proc. of the Modeling and Optimization in Mobile, Ad Hoc and Wireless networks Workshop (WiOpt'03), Mar. 2003.

[21] E. Telatar, "Capacity of multi-antenna gaussian channels," in Europ. Trans. Telecommunications, Nov. 1997.

[22] I. Maric and R. Yates, Efficient Multihop Broadcast for Wideband Systems, Book chapter: multiantenna channels: capacity, coding and signal processing, DIMACS workshop on signal processing for wireless transmission ed., G. J. Foschini and S. Verdu, Eds. American Mathematical Society, Oct. 2002, vol. 62.

[23] I. F. Akyildiz, W. Su, Y. Sankarasubramaniam, and E. Cayirci, "A survey on sensor networks," IEEE Communications Magazine, pp. 102-114, Aug. 2002.

[24] R. G. Gallager, P. A. Humblet, and P. M. Spira, "A distributed algorithm for minimum-weight spanning trees," ACM Trans. on Programming Languages and Systems, vol. 5, no. 1, pp. 66-77, Jan. 1983.

[25] P. A. Humblet, "A distributed algorithm for minimum weight directed spanning trees," IEEE Trans. on Communications, vol. 31, no. 6, June 1983.

[26] J. Cartigny, D. Simplot, and I. Stojmenovic, "Localized minimum-energy broadcasting in ad-hoc networks," in IEEE INFOCOM 2003, Apr. 2003.

[27] J. Wieselthier, G. Nguyen, and A. Ephremides, "The energy efficiency of distributed algorithms for broadcasting in ad hoc networks," in IEEE 5th International Symposium on Wireless Personal Multimedia Communications (WPMC), Oct. 2002, pp. 499-503.

[28] T. Cover and J. Thomas, Elements of Information Theory. John Wiley Sons, Inc., 1991.

[29] S. Verdu, G. Caire, and D. Tuninetti, "Is TDMA optimal in the low power regime?" in Proc. of International Symposium on Information Theory (ISIT'02), July 2002.

[30] P. Gupta and P. R. Kumar, "Critical power for asymptotic connectivity in wireless networks," Stochastic Analysis, Control, Optimization and Applications: A Volume in Honor of W.H. Fleming, 1998.

[31] N. Li and C. Hou, "BLMST: a scalable, power efficient broadcast algorithm for wireless sensor networks," in IEEE INFOCOM 2004, submitted.

[32] S. Narayanaswamy, V. Kawadia, R. S. Sreenivas, and P. R. Kumar, "Power control in ad-hoc networks: Theory, architecture, algorithm and implementation of the COMPOW protocol," in European Wireless 2002, Feb. 2002, pp. 156-162.

[33] S. Narayanaswamy, V. K. R. S. Sreenivas, and P. R. Kumar, "Power control in ad-hoc networks: Theory, architecture, algorithm and implementation of the COMPOW protocol," in Proc. of European Wireless 2002, Feb. 2002, pp. 179-186.

[34] Y. Hong and A. Scaglione, "Energy-efficient broadcasting with cooperative transmission in wireless sensory ad hoc networks," in Proc. of Allerton Conference, Oct. 2003.

[35] R. Knopp and P. A. Humblet, "Information capacity and power control in single-cell multiuser communications," in Proc. of International Conference on Communications (ICC'95), June 1995, pp. 331-335.

[36] M. Grossglauser and D. Tse, "Mobility increases the capacity of adhoc wireless networks," IEEE/ACM Transactions on Networking, vol. 10 , no. 4, pp. 477-486, Aug. 2002. 
[37] R. Frenkiel, B. R. Badrinath, J. Borras, and R. Yates, "The infostations challenge: Balancing cost and ubiquity in delivering wireless data," in Preprint, 1999.

[38] R. Knopp and P. A. Humblet, "Multiple-accessing over frequencyselective fading channels," in 6 th IEEE International Symposium on Personal, Indoor and Mobile Radio Communications (PIMRC'95), Sept. 1995, pp. 1326-1330.

\begin{tabular}{|c|} 
\\
\\
PLACE \\
PHOTO \\
HERE \\
\end{tabular}

Ivana Maric received the B.S. in electrical engineering from University of Novi Sad, Yugoslavia in 1995 and the M.S. in electrical engineering from Rutgers University, Piscataway in 2000. She is a currently working toward the $\mathrm{Ph} . \mathrm{D}$. degree in Wireless Information Network Laboratory (WINLAB) at the same university. She was a summer intern in AT\&T Research Labs, Red Bank, NJ in 1998. Her research interest include network information theory, wireless ad hoc networks and more specifically, wireless relay channels and cooperative broadcast.

\begin{tabular}{|c|} 
\\
\\
PLACE \\
PHOTO \\
HERE \\
\end{tabular}

Roy D. Yates received the B.S.E. degree in 1983 from Princeton University, and the S.M. and Ph.D. degrees in 1986 and 1990 from M.I.T., all in Electrical Engineering. Since 1990, he has been with the Wireless Information Networks Laboratory (WIN$\mathrm{LAB}$ ) and the ECE department at Rutgers University. Presently, he is an Associate Director of WINLAB and a Professor in the ECE Dept. He is a coauthor (with David Goodman) of the text Probability and Stochastic Processes: A Friendly Introduction for Electrical and Computer Engineers published by John Wiley and Sons. Roy Yates is a co-recepient of the 2003 IEEE Marconi Paper Prize Award in Wireless Communications. His research interests include power control, interference suppression and spectrum regulation. 